\title{
Efficiency Enhancement of Solar Cell Using Metamaterials
}

Dena M. El-Amassi ${ }^{1}$, Hala J. El-Khozondar ${ }^{2}$, Mohammed M. Shabat ${ }^{3}$

Research Article

\author{
${ }^{1}$ Research Assistant, Islamic University of Gaza, Gaza, Palestine. \\ ${ }^{2}$ Electrical Engineering department, Islamic University of Gaza, Gaza, Palestine. \\ ${ }^{3}$ Physics Department, Islamic University of Gaza, Gaza, Palestine.
}

\begin{abstract}
In this work, Photovoltaic (PV) cell model with high absorption efficiency is introduced. The proposed four layer PV cell model consists of antireflection coating (ARC) layer on glass based and covered by metamaterial (MTM) bounded by air. The effect of the refractive index of MTM as well as the effect of the incident angle on the reflectance are considered. Results showed that the reflectance of the PV can be controlled by changing the reflectance index of MTM as well changing the incident angle.
\end{abstract}

Keywords: Antireflection coating (ARC); Metamaterials; Solar Cell; Optimization; Reflectance; PV efficiency.

\section{*Corresponding Author:}

Hala J. El-Khozondar,

Electrical Engineering department, Islamic University of Gaza, Gaza, Palestine.

E-mail: hkhozondar@iugaza.edu

Received: April 28, 2015

Accepted: June 10, 2015

Published: June 11, 2015

Citation: Dena M. El-Amassi, Hala J. El-Khozondar, Mohammed M. Shabat (2015) Efficiency Enhancement of Solar Cell Using Metamaterials. Int J Nano Stud Technol. 4(2), 84-87. doi: http://dx.doi. org/10.19070/2167-8685-1500016

Copyright: Hala J. El-Khozondar ${ }^{\circ} 2015$ This is an open-access article distributed under the terms of the Creative Commons Attribution License, which permits unrestricted use, distribution and reproduction in any medium, provided the original author and source are credited.

\section{Introduction}

Renewable energy known as environmentally friendly attracted a lot of attention due to the energy crises especially in the third world. Solar energy is the most promising among other sources of renewable energy because of its abundance in nature and cleanness. The main component in the system is the PV cell. For a PV cell to work appropriately its absorption to sun light must be optimized. Different works have been establish to reach the optimal values for light absorption by PV cell with maintaining reasonable cost of the cell.

These studies focused in using antireflection coating (ARC) to improve the internal structure of the PV cell [1]. The simplest structure is achieved by using a single ARC on a substrate. Different materials are used for $\mathrm{ARC}$ as in [2-5]. $\mathrm{SiN}_{\mathrm{x}}$ is the mostly used as ARC because it has high refractive index value that can varied by changing deposition paramters [6]. A more complex PV cell that lead to mimimum reflecance at wider range of wavelengths is achieved by using double ARC with different materials. Examples of double layer ACR is found in [6-9]. Beye [10] proposed a double ARC solar cell with same materials of $\mathrm{SiN}_{x}$.

In his work, the minimum reflectance is achieved below $5 \%$. In this work, we poposed a solar cell waveguide structure model with even lower reflectance that reach as low as $1 \%$ using double layer one of them is ARC and the other is metamaterial (MTM). Metamaterial is a noval material fabracted at laboratory with negative refractive index. They assume different refractive index by changing certain parameter as in $[11,12]$. Next section is dedicated to introuce the proposed PV cell. Section 3 presents the results and discusion. Finally, the conclusion is given in section 4 .

\section{Proposed Solar Cell (PV)}

Figure 1 exhibits the structure of the proposed solar cell. In the structure, the PV cell consists of $\mathrm{SiN}_{\mathrm{x}}$ thin film deposited on glass and covered by MTMs bounded by air. The refractive indices as denoted in Figure 1 are $\mathrm{n}_{0}, \mathrm{n}_{1}, \mathrm{n}_{2}$, and $\mathrm{n}_{\mathrm{s}}$ for air, MTMs, SiN and glass respectively. MTM layer thickness is $d_{1}$ while the film thickness is $\mathrm{d}_{2}$. The light is assumed to shine on the PV at oblique incidence with different incidence angle $\left(\theta_{0}\right)$.

For oblique incidence, the optical admittance for the $\mathrm{k}^{\text {th }}$ layer including negative index materials is derived in the paper of $[13,14]$ for both transverse electric field polarization (TE) and transvers magnetic field polarization (TM) as follows:

$$
\gamma_{k}^{T E}=\frac{1}{\eta_{0} \mu_{k}} n_{k} \cos \theta_{k}
$$




$$
\gamma_{k}^{T M}=\frac{1}{\eta_{0} \mu_{k}} \frac{n_{k}}{\cos \theta_{k}}
$$

Where $\theta_{k}$ is related to $\theta_{0}$ by Snell's law

$$
n_{0} \sin \theta_{0}=n_{k} \sin \theta_{k} \quad ; k=1,2, \ldots m
$$

The $2 \times 2$ transfer matrix that relates the field components at two successive boundaries (e.g. a and b) is defined as follows:

$$
M_{k}=\left[\begin{array}{ll}
m_{11} & m_{12} \\
m_{21} & m_{22}
\end{array}\right]=\left[\begin{array}{cc}
\cos \left(\delta_{k}\right) & \frac{i \sin \left(\delta_{k}\right)}{\gamma_{k}} \\
i \gamma_{k} \sin \left(\delta_{k}\right) & \cos \left(\delta_{k}\right)
\end{array}\right]
$$

$$
\left[\begin{array}{c}
E_{a} \\
B_{a}
\end{array}\right]=M_{k}\left[\begin{array}{l}
E_{b} \\
B_{b}
\end{array}\right]
$$

where $\delta_{k}=2 \pi n_{k} \mathrm{~d}_{k} \theta_{k} / \lambda$ is the phase difference. For $\mathrm{m}$ layers, the overall transfer matrix $\left(M_{T}\right)$ is defined in terms of individual matrix as follows:

$$
M_{T}=M_{1} M_{2} \ldots M_{m}=\prod_{k=1}^{m} M_{k}
$$

Then the reflectance can be derived from equation (5) and (6). The reflectance $\mathrm{R}=|r|^{2}$ where $r$ is the reflection coefficient defined as:

$$
r=\frac{E_{r 1}}{E_{0}}
$$

Following $[13,14]$, the reflectance for our system is derived for both TE and TM polarization as follows.

For TE the reflectance $\mathrm{R}^{\mathrm{TE}}$ is

$$
\begin{gathered}
n_{0}^{2} \cos \left(\theta_{0}\right)\left[\cos \left(\delta_{1}\right) \cos \left(\delta_{2}\right)-\frac{n_{2} \cos \left(\theta_{2}\right)}{n_{1} \cos \left(\theta_{1}\right)} \sin \left(\delta_{1}\right) \sin \left(\delta_{2}\right)\right]^{2} \\
+\left[n_{0} \cos \left(\theta_{0}\right) n_{s} \cos \left(\theta_{s}\right)\right]^{2}\left[\frac{\cos \left(\delta_{1}\right) \sin \left(\delta_{2}\right)}{n_{2} \cos \left(\theta_{2}\right)}+\frac{\sin \left(\delta_{1}\right) \cos \left(\delta_{2}\right)}{n_{1} \cos \left(\theta_{1}\right)}\right]^{2} \\
+\left[n_{1} \cos \left(\theta_{1}\right) \sin \left(\delta_{1}\right) \cos \left(\delta_{2}\right)+n_{2} \cos \left(\theta_{2}\right) \cos \left(\delta_{1}\right) \sin \left(\delta_{2}\right)\right]^{2} \\
R^{T E}=\frac{+n_{s}^{2} \cos { }^{2}\left(\theta_{s}\right)\left[\cos \left(\delta_{1}\right) \cos \left(\delta_{2}\right)-\frac{n_{1} \cos \left(\theta_{1}\right)}{n_{2} \cos \left(\theta_{2}\right)} \sin \left(\delta_{1}\right) \sin \left(\delta_{2}\right)\right]^{2}-2 n_{0} \cos \left(\theta_{0}\right) n_{s} \cos \left(\theta_{s}\right)}{n_{0}^{2} \cos \left(\theta_{0}\right)\left[\cos \left(\delta_{1}\right) \cos \left(\delta_{2}\right)-\frac{n_{2} \cos \left(\theta_{2}\right)}{n_{1} \cos \left(\theta_{1}\right)} \sin \left(\delta_{1}\right) \sin \left(\delta_{2}\right)\right]^{2}} \\
+\left[n_{0} \cos \left(\theta_{0}\right) n_{s} \cos \left(\theta_{s}\right)\right]^{2}\left[\frac{\cos \left(\delta_{\delta_{1}}\right) \sin \left(\delta_{2}\right)}{n_{2} \cos \left(\theta_{2}\right)}+\frac{\sin \left(\delta_{1}\right) \cos \left(\delta_{2}\right)}{n_{1} \cos \left(\theta_{1}\right)}\right]^{2} \\
+\left[n_{1} \cos \left(\theta_{1}\right) \sin \left(\delta_{1}\right) \cos \left(\delta_{2}\right)+n_{2} \cos \left(\theta_{2}\right) \cos \left(\delta_{\delta^{\prime}}\right) \sin \left(\delta_{2}\right)\right]^{2} \\
+n_{s}^{2} \cos { }^{2}\left(\theta_{s}\right)\left[\cos \left(\delta_{1}\right) \cos \left(\delta_{2}\right)-\frac{n_{1} \cos \left(\theta_{1}\right)}{n_{2} \cos \left(\theta_{2}\right)} \sin \left(\delta_{1}\right) \sin \left(\delta_{2}\right)\right]^{2}+2 n_{0} \cos \left(\theta_{0}\right) n_{s} \cos \left(\theta_{s}\right)
\end{gathered}
$$

and for TM the $R^{T M}$ is

$$
\begin{gathered}
n_{0}^{2} \cos { }^{2}\left(\theta_{0}\right)\left[\cos \left(\delta_{1}\right) \cos \left(\delta_{2}\right)-\frac{n_{2} \cos \left(\theta_{2}\right)}{n_{1} \cos \left(\theta_{1}\right)} \sin \left(\delta_{1}\right) \sin \left(\delta_{2}\right)\right]^{2} \\
+\left[n_{0} \cos \left(\theta_{0}\right) n_{s} \cos \left(\theta_{s}\right)\right]^{2}\left[\frac{\cos \left(\theta_{2}\right) \cos \left(\delta_{1}\right) \sin \left(\delta_{2}\right)}{n_{2}}+\frac{\cos \left(\theta_{1}\right) \sin \left(\delta_{1}\right) \cos \left(\delta_{2}\right)}{n_{1}}\right]^{2} \\
+\left[\frac{n_{1} \sin \left(\delta_{1}\right) \cos \left(\delta_{2}\right)}{\cos \left(\theta_{1}\right)}+\frac{n_{2} \cos \left(\delta_{1}\right) \sin \left(\delta_{2}\right)}{\cos \left(\theta_{2}\right)}\right]^{2} \\
R^{T M}=\frac{+n_{s}^{2} \cos ^{2}\left(\theta_{s}\right)\left[\cos \left(\delta_{1}\right) \cos \left(\delta_{2}\right)-\frac{n_{1} \cos \left(\theta_{1}\right)}{n_{2} \cos \left(\theta_{2}\right)} \sin \left(\delta_{1}\right) \sin \left(\delta_{2}\right)\right]^{2}-2 n_{0} \cos \left(\theta_{0}\right) n_{s} \cos \left(\theta_{s}\right)}{n_{0}^{2} \cos { }^{2}\left(\theta_{0}\right)\left[\cos \left(\delta_{1}\right) \cos \left(\delta_{2}\right)-\frac{n_{2} \cos \left(\theta_{2}\right)}{n_{1} \cos \left(\theta_{1}\right)} \sin \left(\delta_{1}\right) \sin \left(\delta_{2}\right)\right]^{2}} \\
+\left[n_{0} \cos \left(\theta_{0}\right) n_{s} \cos \left(\theta_{s}\right)\right]^{2}\left[\frac{\cos \left(\theta_{2}\right) \cos \left(\delta_{1}\right) \sin \left(\delta_{2}\right)}{\left.n_{2}\right)}+\frac{\cos \left(\theta_{1}\right) \sin \left(\delta_{1}\right) \cos \left(\delta_{2}\right)}{n_{1}}\right]^{2} \\
+\left[\frac{n_{1} \sin \left(\delta_{1}\right) \cos \left(\delta_{2}\right)}{\cos \left(\theta_{1}\right)}+\frac{n_{2} \cos \left(\delta_{1}\right) \sin \left(\delta_{2}\right)}{\cos \left(\delta_{2}\right)}\right]^{2} \\
+n_{s}^{2} \cos { }^{2}\left(\theta_{s}\right)\left[\cos \left(\delta_{1}\right) \cos \left(\delta_{2}\right)-\frac{n_{1} \cos \left(\theta_{2}\right)}{n_{2} \cos \left(\theta_{1}\right)} \sin \left(\delta_{1}\right) \sin \left(\delta_{2}\right)\right]^{2}+2 n_{0} \cos \left(\theta_{0}\right) n_{s} \cos \left(\theta_{s}\right)
\end{gathered}
$$

The total reflectance $R$ for the solar cell is defined as the average of both values $R^{T E}$ and $R^{T M}$ :

$$
R=\frac{R^{T E}+R^{T M}}{2}
$$

The Total reflectance (equation (10)) is solved using the software Maple 13 to verify the characteristics of the proposed PV cell.

\section{Results and Discussion}

The structure under consideration has film consisting of $\mathrm{SiN}_{\mathrm{x}}$ on glass substrate with $n_{s}=1.47$, and covered by negative refractive index MTM bonded from above by air with $\mathrm{n}_{0}=1$. The thicknesses $\mathrm{d}_{1}$ and $\mathrm{d}_{2}$ are taken to be equal the quarter wavelength at each media $\left(\mathrm{d}_{\mathrm{m}}=\lambda_{\mathrm{m}} / 4\right)$, where $\lambda_{\mathrm{m}}=\lambda / \mathrm{n}_{\mathrm{m}}$ and $\mathrm{n}_{\mathrm{m}}$ is the refractive index for each media. Usually the wavelength $\lambda$ is chosen close to the peak of the solar spectrum $(\lambda=600 \mathrm{~nm})$. The spectral response of $\mathrm{SiN}_{\mathrm{x}}$ goes from 300-1200nm [10]. Thus, this range is taken to limit the spectrum of the incident light. In our calculation, we consider first normal incidence with $n_{1}=-2$ and vary $n_{2}$ to achieve optimal value of $n_{2}$ at which the reflectance equals zero. Figure 2 exhibits the total reflectance $R$ as calculated from equation (10) using Maple 13. In the calculation, we let $\mathrm{n}_{2}$ assume the following values: 2.5, 2.4, 2.3, and 2.2 as indicated in Figure 2. We notice that the spectral takes zero value when $\mathrm{n}_{2}=2.4$ and all other curves maintain a reflectance lower than $1 \%$. Comparing our result with one or two ARC layers in Beye calculations [10], we obtain a better result since his system maintained reflectance fewer than $5 \%$.

To further check the optimal structure, we let $\mathrm{n}_{2}=2.4$ and vary $n_{1}$ as in Figure 3. We realize that the optimal value of $n_{1}=-2$. Moreover, with excluding $n_{1}=-1.6$, we found that reflectance at $600 \mathrm{~nm}$ below $1.5 \%$ which lower than the values obtained by [10].

Figure 4 displays the results for the proposed PV cell at $\mathrm{n}_{2}=2.4$ and $n_{1}=-2$ with oblique incidence. We notice that for low angles $\left(\theta_{0}=0^{\circ}, 15^{\circ}, 30^{\circ}\right.$ and $\left.45^{\circ}\right)$ the reflectance is below $4.5 \%$ almost in the whole spectrum and for $\theta_{0}=60^{\circ}$ the reflectance is below $8 \%$ for the whole spectrum. These results are better than the result obtained by Beye [10]. 
Figure 1. The schematic of the proposed nanostructure Solar Cell.

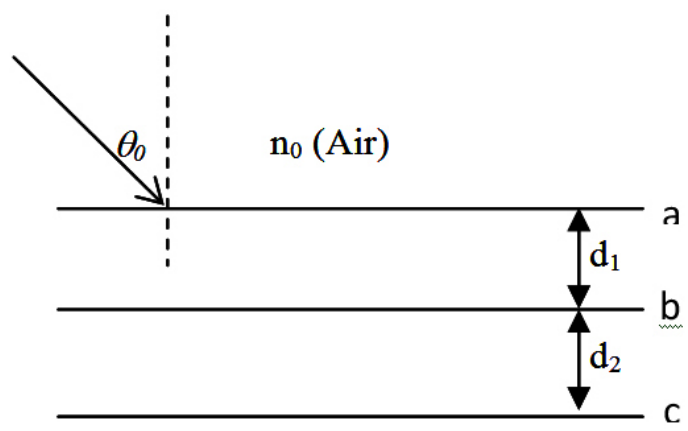

Figure 2. The reflectance calculated for $n_{1}=-2$, under normal incidence.

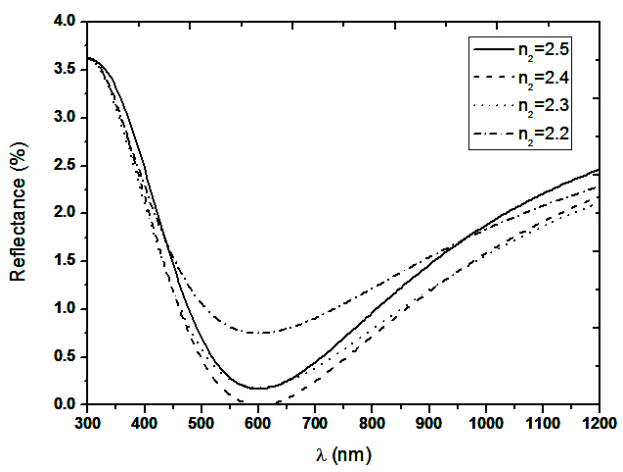

Figure 3. The reflectance for $n_{2}=2$ and under normal incidence.

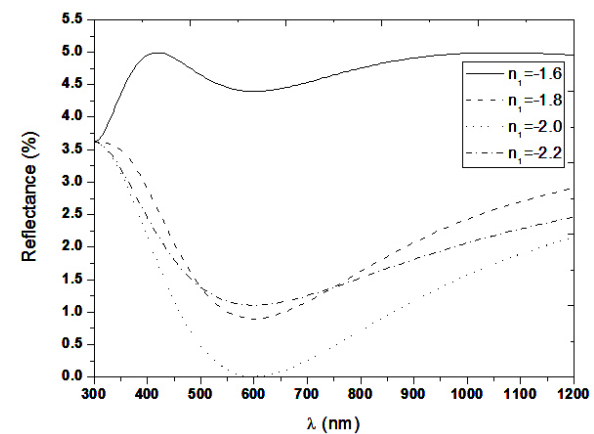

Figure 4. The reflectance for oblique incidence when $n_{1}=-2$ and $n_{2}=2.4$.

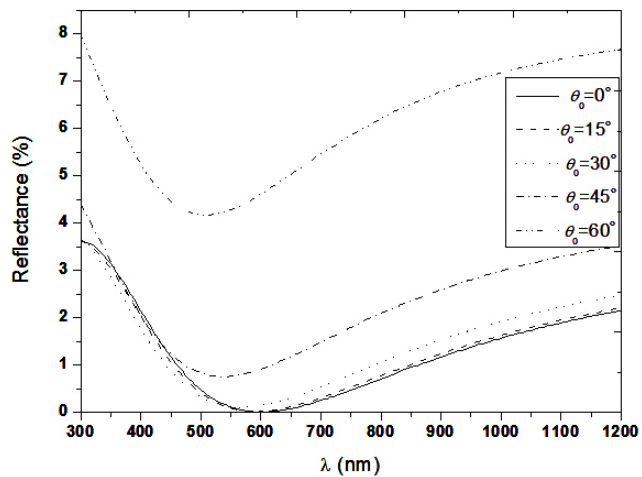

\section{Conclusion}

In this work, we proposed double layer PV cell with different materials where one layer consists of MTM and the other is ARC made of SiN. The double layers are bounded from below by glass and coved by air. The average reflectance is numerically solved using maple 13. The numerical calculations are obtained under different condition; by varying the MTM parameters, or varying $\mathrm{SiN}_{\mathrm{x}}$ refractive index, or at different incident angles. Results show that the average reflectance reached its minimum when $\mathrm{n}_{1}=-2, \mathrm{n}_{2}=2.4$ and for normal incidence. Moreover, the average reflectance under different conditions low compared to other proposed structures. The results are useful to improve the efficiency of PV cell. 


\section{References}

[1]. Rancourt JD (1996) Optical thin films: User's handbook, Washington:SPIE Optical Engineering Press.

[2]. Bouhafs D, Moussi A, Chikouche A, Ruiz JM (1998) Design and simulation of antireflection coating systems for optoelectronic devices: Application to silicon solar cells. Solar Energy Materials and Solar Cells 52: 79-93.

[3]. Barshilia HC, Selvakumar N, Rajam KS (2006) TiAIN/TiAlON/Si3N4 tandem absorber for high temperatute solar selective applications, Appl. Phys. Lett., 89: 191909-3.

[4]. Lee HC, Su YK, Chuang WK, Lin JC, Huang KC et.al, (2010) Discussion on electrical characteristics of $\mathrm{i}-\mathrm{In} 0.13 \mathrm{Ga} 0.87 \mathrm{~N}$ p-i-n photovoltaics by using a single/multi-antireflection layer. Solar Energy Materials and Solar Cells 94(7):1259-1262.

[5]. Sopori B (2005) Dielectric films for Si solar cell applications.J. Electron Mater 34: $564-570$

[6]. Lee Y, Gong D, Balaji N, Lee Y, Yi J (2012) Stability of SiNx/SiNx double stack antireflection coating for single crystalline silicon solar cells. Nanoscale Res. Lett. 7: 50-56.

[7]. Alemu A, Freundlich A, Badi N, Boney C, Bensaoula A (2010) Low temperature deposited boron nitride thin films for a robust antireflection coating of solar cells. Solar Energy Mater, Solar Cells. 94(5): 921-923.

[8]. Taguchi H, Soga T, Jimbo T (2003) Fabrication of GaAs/Si tandem solar cell by epitaxial lift-off technique. Japanese Journal of Applied Physics Part 2-letters 42 L1419-1421.

[9]. Valdivia E, Desfonds E, Masson D, Fafard S, Carlson A et.al (2008) Optimization of antireflection coating design for multi-junction solar cells and concentrator systems.Proceedings of the society of Photo-Optical Instrumentation Engineers 7099.

[10]. Beye M, Faye ME, Ndiaye A, Ndiaye F, Maiga AS (2013) Optimization of SiNx Single and Double Layer ARC for Silicon Thin Film Solar Cells on Glass. Research Journal of Applied Sciences, Engineering and Technology 6(3): 412-416

[11]. El-Khozondar HJ, El-Khozondar RF, Zouhdi S (2015) Tunable MTMs consists of a single-walled nanotube thin film waveguide covered by nonlinear cladding.Applied Physics A 119: 451-453.

[12]. Werner DH, Kwon D, Khoo I, Kildishev AV, Shalaev VM (2011) Liquid crystal clad near-infrared metamaterials with tunable negative-zero positive refractive indices. Optics Express 15(6): 3342-3347.

[13]. Al-Turk S (2011) Analytic optimization modeling of anti-reflection coating for solar cells, Master thesis, MacMaster University: Open Dissertations and theses.

[14]. Lequime M, Gralak B, Guenneau S, Zerrad M, Amra C (2013) Optical properties of multilayer optics including negative index materials. PhysicsOptics 1-9. 